\title{
Molecular Dynamics Simulation of Aqueous Solutions of 26-Unit Segments of p(NIPAAm) and of p(NIPAAm) "Doped" with Amino Acid Based Comonomers
}

\author{
Fabrizio Gangemi, ${ }^{*},+, *$ Giovanna Longhi, ${ }^{\dagger, *}$ Sergio Abbate, ${ }^{\dagger, *}$ France Lebon,,,+ \\ Roberto Cordone, ${ }^{\S}$ Gian Paolo Ghilardi, ${ }^{\S}$ and Sandro L. Fornilit, \\ Dipartimento di Scienze Biomediche e Biotecnologie, Università di Brescia, Viale Europa 11, 25123 Brescia, \\ Italy, Consorzio Nazionale Interuniversitario per le Scienze Fisiche della Materia (CNISM), Via della Vasca \\ Navale, 84 - 00146 Roma, Italy, and Dipartimento di Tecnologie dell'Informazione, Università di Milano, \\ via Bramante 65, 26013 Crema, Italy
}

Received: April 23, 2008; Revised Manuscript Received: July 16, 2008

\begin{abstract}
We have performed 75-ns molecular dynamics (MD) simulations of aqueous solutions of a 26-unit NIPAAm oligomer at two temperatures, 302 and $315 \mathrm{~K}$, below and above the experimentally determined lower critical solution temperature (LCST) of p(NIPAAm). We have been able to show that at $315 \mathrm{~K}$ the oligomer assumes a compact form, while it keeps a more extended form at $302 \mathrm{~K}$. A similar behavior has been demonstrated for a similar NIPAAm oligomer, where two units had been substituted by methacryloyl- $l$-valine (MAVA) comonomers, one of them being charged and one neutral. For another analogous oligomer, where the same units had been substituted by methacryloyl-l-leucine (MALEU) comonomers, no transition from the extended to the more compact conformation has been found within the same simulation time. Statistical analysis of the trajectories indicates that this transition is related to the dynamics of the oligomer backbone, and to the formation of intramolecular hydrogen bonds and water-bridges between distant units of the solute. In the MAVA case, we have also evidenced an important role of the neutral MAVA comonomer in stabilizing the compact coiled structure. In the MALEU case, the corresponding comonomer is not equally efficacious and, possibly, is even hindering the readjustment of the oligomer backbone. Finally the self-diffusion coefficient of water molecules surrounding the oligomers at the two temperatures for selected relevant times is observed to characteristically depend on the distance from the solute molecules.
\end{abstract}

\section{Introduction}

The linear form of poly( $N$-isopropylacrylamide) (p(NIPAAm)) has been studied for several years, due to its numerous applicative potentialities ${ }^{1-4}$ related to the interesting phase transition phenomenon it undergoes in water solution. Indeed the increase of temperature brings about higher turbidity in the solution and eventually leads to precipitation. This occurs at ca. $32{ }^{\circ} \mathrm{C}$ allowing one to define the lower critical solution temperature (LCST). ${ }^{5}$ In the latter respect several works have been presented, and we particularly refer to the light scattering (LS) experiments by Rička et al. ${ }^{6}$ and by $\mathrm{Wu}$ and Wang, ${ }^{7}$ aiming at identifying purely intrachain factors determining the phase transition: the cited authors have done so by either isolating the p(NIPAAm) chains from each other with surfactant molecules or by looking at very dilute and monodispersed long p(NIPAAm) chains. Some of us have contributed to this field, by studying via LS and circular dichroism (CD) 10:1 copolymers of NIPAAm and $N$-methacryloyl-l-valine (MAVA) or $\mathrm{N}$ methacryloyl- $l$-leucine (MALEU) ${ }^{8}$ (see Figure 1 ). The presence of such amino acid derived comonomers on one hand confers $\mathrm{pH}$-sensitivity to the copolymer and also hinders precipitation when temperature is increased. It also changes the LCST and the temperature width of the phase transition interval. In the latter study, intra- and interchain effects were both present and

\footnotetext{
* Corresponding author.

$\uparrow$ Dipartimento di Scienze Biomediche e Biotecnologie, Università di Brescia.

$¥$ Consorzio Nazionale Interuniversitario per le Scienze Fisiche della Materia (CNISM).

$\S$ Dipartimento di Tecnologie dell'Informazione, Università di Milano.
}

were distinguished, up to a certain point, by simultaneously studying the total scattering and the dynamic $\mathrm{LS}^{8}$ in particular, relevant differences between the two copolymers had been pointed out (we need to remember that several authors have contributed to this field, among them Casolaro ${ }^{9}$ and Winnik et al. ${ }^{10}$ ).

Another study of our own ${ }^{11}$ conducted by means of molecular dynamics (MD) simulations on a 50-unit oligomer of NIPAAm allowed to evidence at the microscopic scale a relatively known fact, that is to say that water has a crucial role in modulating the conformational changes of $\mathrm{p}$ (NIPAAm). ${ }^{5}$ Indeed we found that below and above LCST the model oligomer exhibits different gyration radii ( $c f$. Figure 3 in ref 11) and water organizes itself differently close and away from the oligomer ( $c f$. Figure 5 in ref 11). We wish here to extend the study started in ref 11 by making the following forward steps: $(i)$ we considerably increase the simulation length; (ii) besides NIPAAm oligomers, we study here also two oligomers of NIPAAm, one including two MAVA comonomers and the other including two MALEU comonomers, in order to provide some hints to interpret the data of ref 8 and analogous data. To cope with CPU-time requirements, we had to deal with shorter oligomers, i.e., 26-unit segments. Moreover, in order to better compare the properties of the three considered systems, that we call here $\mathrm{oN}$, oNM, and oNL, we have performed MD simulations at two temperatures, 302 and $315 \mathrm{~K}$. These two values are slightly below and above the experimental p(NIPAAm) LCST and the LCST for $\mathrm{p}$ (NIPAAm-co-MAVA) at $\mathrm{pH} 4$; they are both above the LCST for $\mathrm{p}$ (NIPAAm-co-MALEU) at $\mathrm{pH} 4$. At this $\mathrm{pH}$ value the transition is quite sharp. ${ }^{12}$ The initial conformations 


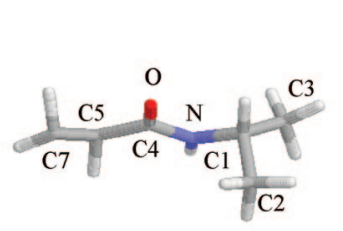

a<smiles>CCC(C)NC(C)C(C)C</smiles>

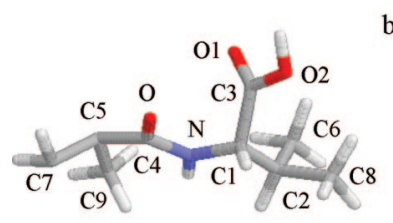

b
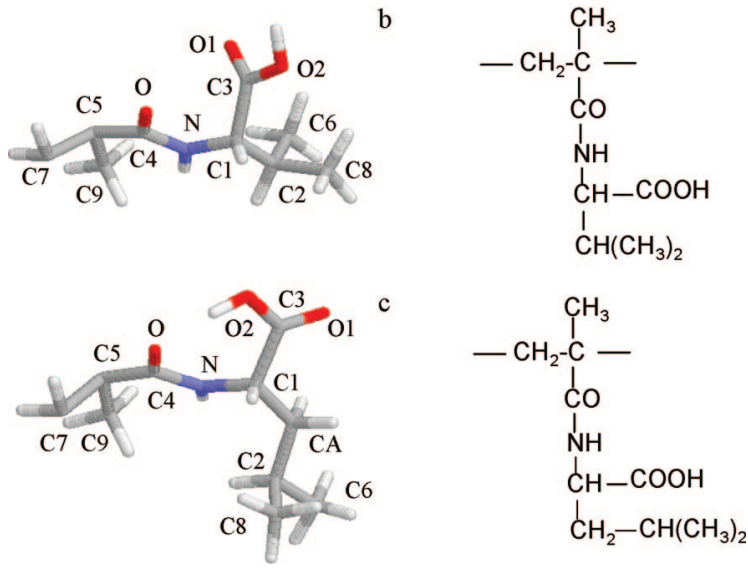

Figure 1. Selected all-trans conformation for the three residues $N$-isopropylacrylamide (NIPAAm (a)) methacryloyl-l-valine (MAVA (b)), and methacryloyl-l-leucine (MALEU (c)), together with the corresponding chemical formulas.

were chosen to be linear for all three oligomers and, as far as possible, identical, as described in the following section.

\section{Computational Methods}

$A b$ initio quantum mechanical calculations were performed to evaluate geometries and atomic charges of the NIPAAm, MAVA, and MALEU monomers. A conformational analysis was carried out for each monomer by means of molecular mechanics methods. ${ }^{13}$ Each conformer geometry was first optimized with GAUSSIAN03 ${ }^{14}$ at the RHF/6-31G* level and the RESP protocol ${ }^{15}$ was then applied to determine the atomic charges. The final values, reported in Table 1, were obtained as an average over conformers weighted by Boltzmann populations at room temperature. In part 1 of the Supporting Information, we provide the Cartesian coordinates for the monomers' geometries employed in the initial conditions of all subsequent calculations.

The oN oligomer (Figure 2a) was "synthesized" by connecting the $\mathrm{C}_{7}$ atom of one unit with the $\mathrm{C}_{5}$ atom of the next unit (see Figure 1) using the LEaP module of AMBER7 suite of programs $;{ }^{16}$ the NIPAAm monomers were randomly set in $d$ and $l$-configurations with respect to $\mathrm{C}_{5}$, obtaining an almost atactic chain (54\% meso ( $d d$ or $l l)$ and $46 \%$ racemic ( $d l$ or $l d$ )
TABLE 1: Charges in Electron Charge Units for the Three Monomers Used in the Simulations ${ }^{a}$

\begin{tabular}{|c|c|c|c|c|c|c|c|}
\hline atom & $\begin{array}{l}\text { NIPAAm } \\
\text { charge }\end{array}$ & atom & $\begin{array}{l}\text { nMAVA } \\
\text { charge }\end{array}$ & $\begin{array}{l}\text { cMAVA } \\
\text { charge }\end{array}$ & atom & $\begin{array}{l}\text { nMALEU } \\
\text { charge }\end{array}$ & $\begin{array}{l}\text { cMALEU } \\
\text { charge }\end{array}$ \\
\hline $\mathrm{N}$ & -0.5498 & $\mathrm{~N}$ & -0.3446 & -0.3321 & $\mathrm{~N}$ & -0.4688 & -0.4454 \\
\hline $\mathrm{H}$ & 0.2928 & $\mathrm{C} 1$ & 0.0607 & -0.0040 & $\mathrm{C} 1$ & 0.0782 & 0.0234 \\
\hline $\mathrm{C} 1$ & 0.3241 & $\mathrm{C} 2$ & 0.0462 & 0.1297 & $\mathrm{C} 2$ & 0.2492 & 0.2401 \\
\hline H1 & 0.0483 & C3 & 0.7078 & 0.7277 & C3 & 0.7219 & 0.7516 \\
\hline $\mathrm{C} 2$ & -0.2838 & C6 & -0.1894 & -0.2140 & C6 & -0.2145 & -0.2261 \\
\hline $\mathrm{H} 21$ & 0.0726 & $\mathrm{C} 8$ & -0.1894 & -0.2140 & $\mathrm{C} 8$ & -0.2145 & -0.2261 \\
\hline $\mathrm{H} 22$ & 0.0726 & $\mathrm{O} 1$ & -0.5788 & 598 & $\mathrm{O} 1$ & -0.5772 & 7692 \\
\hline $\mathrm{H} 23$ & 0.0726 & $\mathrm{O} 2$ & -0.6615 & -0 & $\mathrm{O} 2$ & -0.6547 & -0.7692 \\
\hline C3 & -0.2838 & $\mathrm{C} 4$ & 0.4010 & 0.4647 & $\mathrm{C} 4$ & 0.5071 & 5221 \\
\hline H31 & 0.0726 & $\mathrm{O}$ & -0.5293 & -0.5973 & $\mathrm{O}$ & -0.5387 & -0.6025 \\
\hline H32 & 0.0726 & C7 & 0.0089 & -0.0040 & C7 & 0.0033 & 0.0048 \\
\hline H33 & 0.0726 & C5 & 0.0278 & 0.0280 & C5 & 0.0098 & 0.0263 \\
\hline $\mathrm{O}$ & -0.5703 & C9 & -0.1894 & -0.2140 & C9 & -0.2145 & -0.2261 \\
\hline $\mathrm{C} 4$ & 0.5678 & H21 & 0.0488 & 0.0291 & H21 & -0.0257 & 0.0076 \\
\hline C5 & -0.0367 & H61 & 0.0540 & 0.0441 & H61 & 0.0520 & 0.0406 \\
\hline H5 & 0.0253 & H62 & 0.0540 & 0.0441 & H62 & 0.0520 & 0.0406 \\
\hline C7 & -0.0107 & H63 & 0.0540 & 0.0441 & H63 & 0.0520 & 0.0406 \\
\hline $\mathrm{H} 72$ & 0.0206 & H81 & 0.0540 & 0.0441 & H81 & 0.0520 & 0.0406 \\
\hline \multirow[t]{13}{*}{$\mathrm{H} 73$} & 0.0206 & H82 & 0.0540 & 0.0441 & H82 & 0.0520 & 0.0406 \\
\hline & & H83 & 0.0540 & 0.0441 & H83 & 0.0520 & 0.0406 \\
\hline & & H11 & 0.1122 & 0.0671 & H11 & 0.1160 & 0.0762 \\
\hline & & $\mathrm{H}$ & 0.2537 & 0.2212 & $\mathrm{H}$ & 0.2770 & 0.2530 \\
\hline & & H71 & 0.0286 & 0.0173 & $\mathrm{H} 72$ & 0.0280 & 0.0086 \\
\hline & & $\mathrm{H} 72$ & 0.0286 & 0.0173 & $\mathrm{H} 71$ & 0.0280 & 0.0086 \\
\hline & & H91 & 0.0540 & 0.0441 & H91 & 0.0520 & 0.0406 \\
\hline & & H92 & 0.0540 & 0.0441 & H92 & 0.0520 & 0.0406 \\
\hline & & H93 & 0.0540 & 0.0441 & H93 & 0.0520 & 0.0406 \\
\hline & & $\mathrm{HO} 2$ & 0.4721 & & $\mathrm{HO} 2$ & 0.4636 & \\
\hline & & & & & CA & -0.1647 & -0.0823 \\
\hline & & & & & $\mathrm{HA}$ & 0.0616 & 0.0296 \\
\hline & & & & & HA2 & 0.0616 & 0.0296 \\
\hline
\end{tabular}

${ }^{a}$ In particular, nMAVA is the neutral form of MAVA, shown in Figure 1, while cMAVA is the charged form, obtained by removing atom $\mathrm{HO} 2$ from the neutral one. Similarly, the neutral (nMALEU) and charged (cMALEU) forms of MALEU are reported. Atomic labels are displayed in Figure 1.

diads). The analogous MAVA- and MALEU-based oligomers oNM and oNL were obtained by replacing the NIPAAm units number 9 and 19 with MAVA and MALEU comonomers with their own proper $a b$ initio geometries, respectively, leaving exactly the remaining NIPAAm units in the same initial geometry as oN. The positions of the two comonomers were chosen in such a way that they are separated by ten units, in accordance with the experiments of ref 8, and have approximately the same distance from the oligomer ends. Since the ionization probability at $\mathrm{pH} 4$ (where one observes the sharpest possible transition ${ }^{12}$ ) has been estimated $\mathrm{ca}$. $50 \%$ for these comonomers, ${ }^{8,9}$ ionized and neutral forms have been chosen for unit 9 and 19, respectively. Aqueous solutions were
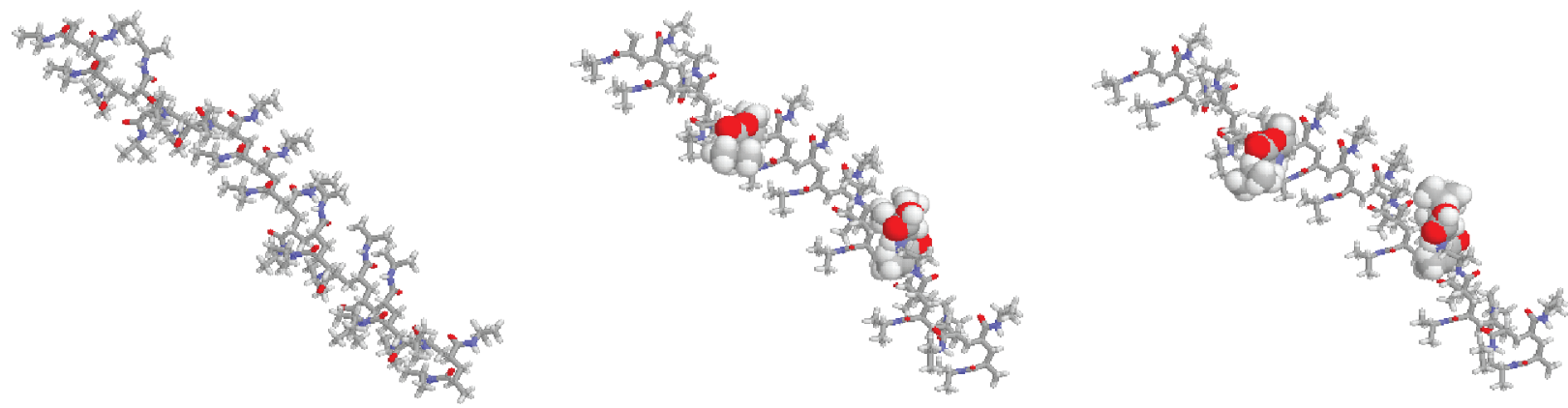

Figure 2. Initial conformations of the 26-unit oligomers: (NIPAAm) $)_{26}$, (NIPAAm) ${ }_{24}-\mathrm{Co}-(\mathrm{MAVA})_{2}$ and $(\mathrm{NIPAAm})_{24}-\mathrm{Co}-(\mathrm{MALEU})_{2}$, which are referred to as oN, oNM, and oNL respectively in the text (from left to right). 
TABLE 2: Water Self-Diffusion Coefficient (in $10^{-5} \mathrm{~cm}^{2} / \mathrm{s}$ ) Calculated for a Sample of 454 TIP3P Water Molecules Alone and for the Water Molecules Surrounding the Three Oligomers oN, oNM and $\mathrm{oNL}^{a}$

\begin{tabular}{ccccc}
\hline $\mathrm{T}(\mathrm{K})$ & Water (TIP3P) & $\mathrm{oN}$ & $\mathrm{oNM}$ & $\mathrm{oNL}$ \\
\hline 298 & $5.5 \pm 0.1$ & & & \\
302 & $5.8 \pm 0.1$ & $5.8 \pm 0.1$ & $5.8 \pm 0.1$ & $5.8 \pm 0.1$ \\
315 & $6.7 \pm 0.1$ & $6.9 \pm 0.1$ & $6.9 \pm 0.1$ & $6.8 \pm 0.1$
\end{tabular}

${ }^{a}$ These values are obtained with a shell radius $r_{h}=30 \AA$ and correspond to the rightmost points on the plots of Figure 13.

generated by surrounding each 26-mer with 3560 TIP3P $\mathrm{P}^{17}$ water molecules and imposing octahedral periodic boundary conditions. The resulting average density during the simulations, turned out to be $0.985 \mathrm{~g} / \mathrm{cm}^{3}$ at $302 \mathrm{~K}$ and 0.974 at $315 \mathrm{~K}$; the resulting average volume of the box of water molecules was $1.13 \cdot 10^{5} \AA^{3}$ at $302 \mathrm{~K}$ and $1.15 \cdot 10^{5} \AA^{3}$ at $315 \mathrm{~K}$. Counterions were added where appropriate to make the systems neutral. The total length of each simulation was $75 \mathrm{~ns}$, following 50-ps NVT and 50-ps NPT equilibration phases. Simulations were performed using the Amber7 SANDER module, ${ }^{16}$ and the AMBER ff03 force field. ${ }^{18}$ The temperature was controlled according to the Berendsen coupling algorithm ${ }^{19}$ with $\tau_{\mathrm{T}}=1 \mathrm{ps} ; \tau_{\mathrm{P}}=1 \mathrm{ps}$ was used for the pressure control at $P=1.0$ bar. A $9-\AA$ cutoff and particle-mesh Ewald (PME) were adopted for the nonbonded interactions. SHAKE ${ }^{19}$ kept constrained the bonds involving hydrogen atoms. The integration step was set to $2 \mathrm{fs}$. Statistical analyses of trajectories were performed using the PTRAJ and CARNAL modules. ${ }^{16}$ Solvent accessible surface area (SASA) was evaluated according to methods outlined in ref 20. Graphic visualization was done with gOpenMol, ${ }^{21} \mathrm{RASMOL}^{22}$ and VMD. ${ }^{23}$ The intramolecular hydrogen bond (HB) definition is similar to that given in ref 24 : donor-acceptor distance $\leq 3.5$ $\AA$, donor-hydrogen acceptor angle $\geq 90^{\circ}$, and hydrogen-acceptor distance $\leq 2.5 \AA .24$

\section{Analysis of the Results and Discussion}

The comparative analysis of MD trajectories yields interesting properties that we wish to examine here. Some properties are more directly attributable to the solute, some others to the solvent, but all of them are inter-related, as we will see, and allow us to develop a coherent picture, at a microscopic scale of detail, of mechanisms underlying structural transitions for the examined systems.

In Figure 3, we report the time evolution of the root mean square distance, $\operatorname{rmsd}(t)$ for the three oligomers oN, oNM and oNL at $302 \mathrm{~K}$ (top panels) and at $315 \mathrm{~K}$ (bottom panels). We are reminded that

$$
\operatorname{rmsd}(t)=\sqrt{\sum_{i=1}^{N} d_{i}^{2}(t) / N}
$$

$N$ being the number of considered solute atoms, in the present case $\mathrm{C}_{5}$ and $\mathrm{C}_{7}$ backbone atoms, and $d_{i}(t)$ the distance of atom $i$ in the configuration at time $t$ with respect to its position in the reference configuration, namely the solute conformation at the end of the equlibration phases. The study of $\operatorname{rmsd}(t)$ helps to monitor conformational changes of the solute. ${ }^{13}$ At $302 \mathrm{~K}$ (which is slightly below LCST for oN and oNM corresponding polymers $^{8}$ ), oN has moderate to high fluctuations around the value of $4 \AA$ during the first $40 \mathrm{~ns}$; from 40 to 75 ns fluctuations increase reaching values close to $10 \AA$, and no "equilibrium" conditions are attained. For oNM and, to a minor extent, for oNL large fluctuations of rmsd are observed at all times. The situation is radically different at $315 \mathrm{~K}$ (which is above LCST for all three corresponding polymers ${ }^{8,12}$ ): indeed for oN, rmsd fluctuates around $4 \AA$ for $20 \mathrm{~ns}$, then undergoes larger jumps between 20 and $45 \mathrm{~ns}$, and reaches a seemingly stable value of $12 \AA$, while fluctuations become smaller. The oNM oligomer has a similar behavior, with the following differences: the large fluctuations leading to a structural change begin at earlier times and the final quite stable value of $14 \AA$ is reached before and does not change up to the end of the simulation. For oNL instead fluctuations are large at all times and no stable structure is reached.

The rmsd time behavior has a suggestive counterpart in the time behavior of the gyration radius, defined as:

$$
R_{G}=\sqrt{\frac{\sum_{i=1}^{N} m_{i}\left(\mathbf{r}_{i}-\mathbf{R}\right)^{2}}{\sum_{i=1}^{N} m_{i}}}
$$

$\mathbf{r}_{i}$ and $m_{i}$ being the position vector and mass of atom $i$ respectively, and $\mathbf{R}$ the position vector of the center of mass of
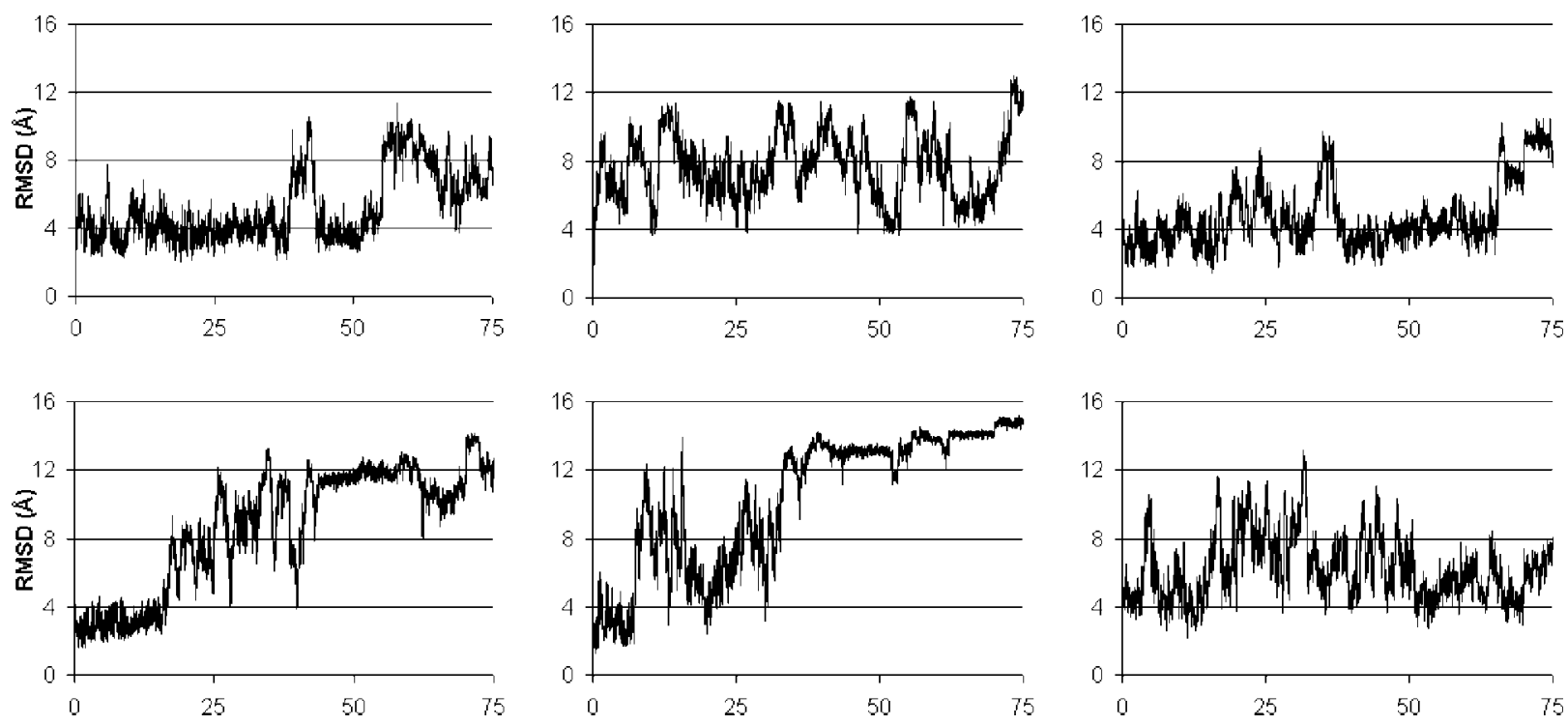

Figure 3. Time evolution of the $\mathrm{C}_{5}$ and $\mathrm{C}_{7}$ backbone atoms root mean square distance (rmsd) for oN, oNM, and oNL (from left to right) at 302 $\mathrm{K}$ (top panels) and $315 \mathrm{~K}$ (lower panels). 

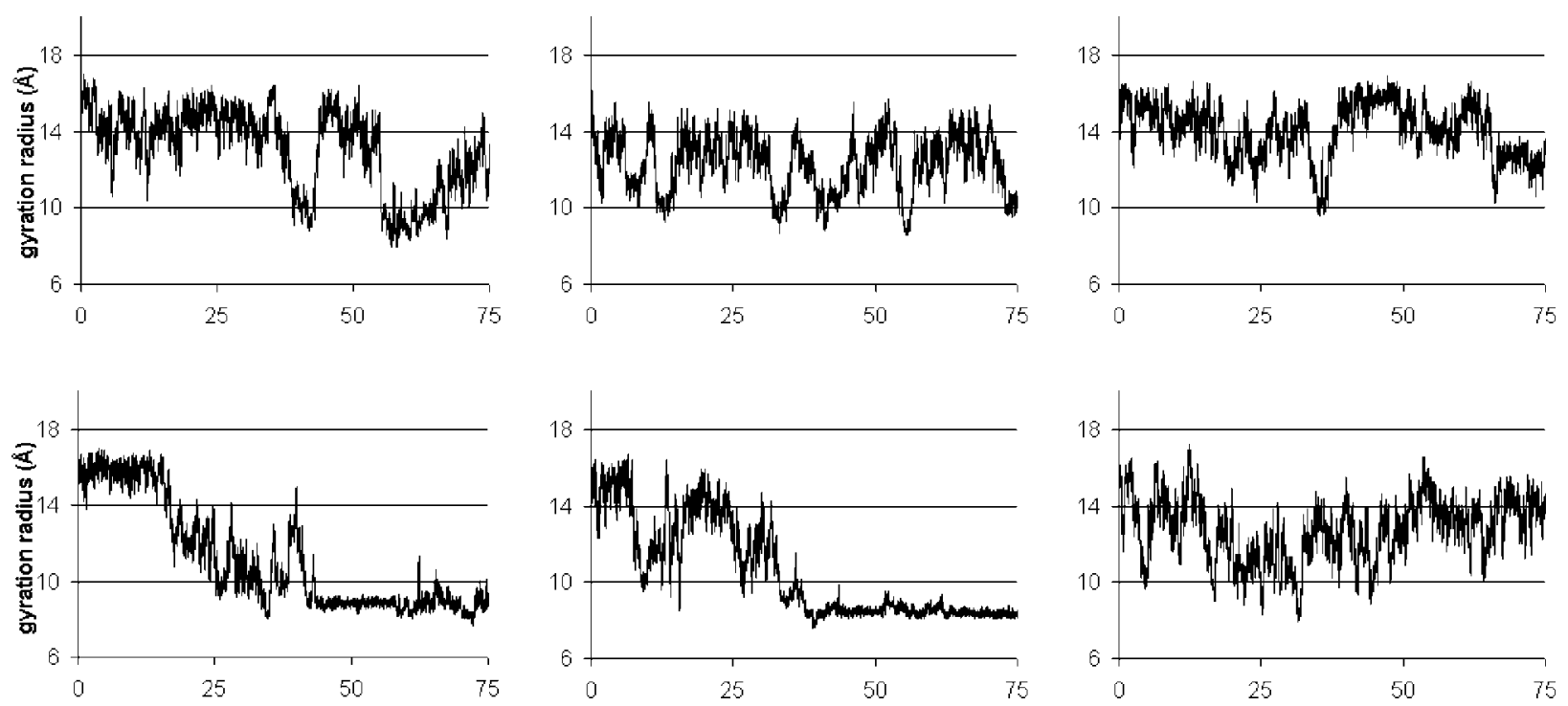

Figure 4. Time evolution of the $\mathrm{C}_{5}$ and $\mathrm{C}_{7}$ backbone atoms gyration radius $R_{G}$, for oN, oNM, and oNL (from left to right) at $302 \mathrm{~K}$ (top panels) and $315 \mathrm{~K}$ (lower panels).
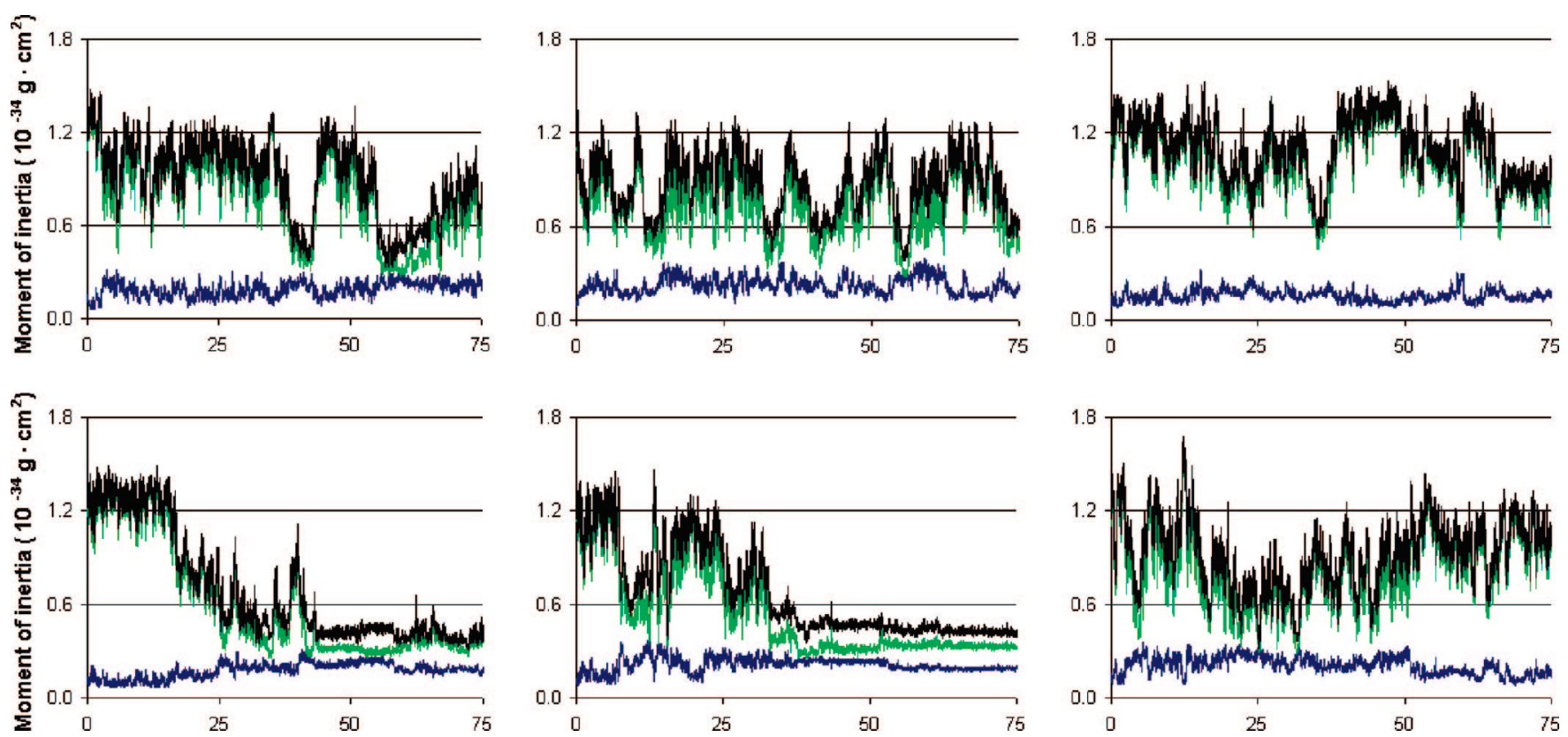

Figure 5. Time evolution of the principal moments of inertia for oN (left), oNM (center), and oNL (right), at $T=302 \mathrm{~K}$ (top) and $T=315 \mathrm{~K}$ (bottom).

the chosen atoms, which in the present case are the $\mathrm{C}_{5}$ and $\mathrm{C}_{7}$ backbone atoms. ${ }^{11}$ In Figure 4 we report $R_{G}$ plots in the same sequence as in Figure 3. The correspondence of the plots of Figures 3 and 4 is remarkable, even though not fully unexpected. The advantage of considering $R_{G}$, is that from Figure 4 we may get an idea of the effective dimensions of the oligomers. Thus we learn that while at $302 \mathrm{~K}$ the three oligomers have an average structure with an $R_{G}$ value that is around $12 \AA$ (with oN decreasing to $c a$. $10-11 \AA$, but showing no stability), at $315 \mathrm{~K}$ $\mathrm{oN}$ and oNM, from about $40 \mathrm{~ns}$ on, attain a compact, closed conformation with an $R_{G}$ value of about $9 \AA$. By direct inspection, using graphical programs, we see that both oN and oNM make rather stable loops connecting distant units via HB and water-bridges. Thus in oN unit 1 is hydrogen-bonded to unit 22, while in oNM units 1,2 , and 3 are very often bonded to unit 19 . oNL instead does not achieve at any time a stable loop, and it rather keeps dimensions more typical of the initial conformation.
Similar information has been obtained by studying the inertia tensor of the oligomer calculated on all atoms $((I))$, whose Cartesian components are defined by:

$$
I_{i j}=\sum_{k=1}^{N} m_{k}\left(\left(\mathbf{r}_{k}-\mathbf{R}\right)^{2} \delta_{i j}-\left(r_{k i}-R_{i}\right)\left(r_{k j}-R_{j}\right)\right)
$$

where $\boldsymbol{R}$ is the position vector of the center of mass of the oligomer. In Figure 5, we report the time evolution of the three eigenvalues of $((I))$, that are usually called principal moments of inertia: two of them, in the initial steps of the MD simulation, i.e. when the oligomers are linear, are almost degenerate and one is much smaller, as expected, since the latter corresponds to the principal axis approximately oriented along the oligomer backbone. When the oligomers coil up, the three eigenvalues coalesce to a common value; still, the time evolution of the two largest principal moments of inertia parallels the time evolution of $R_{G}$ and rmsd in most if not 

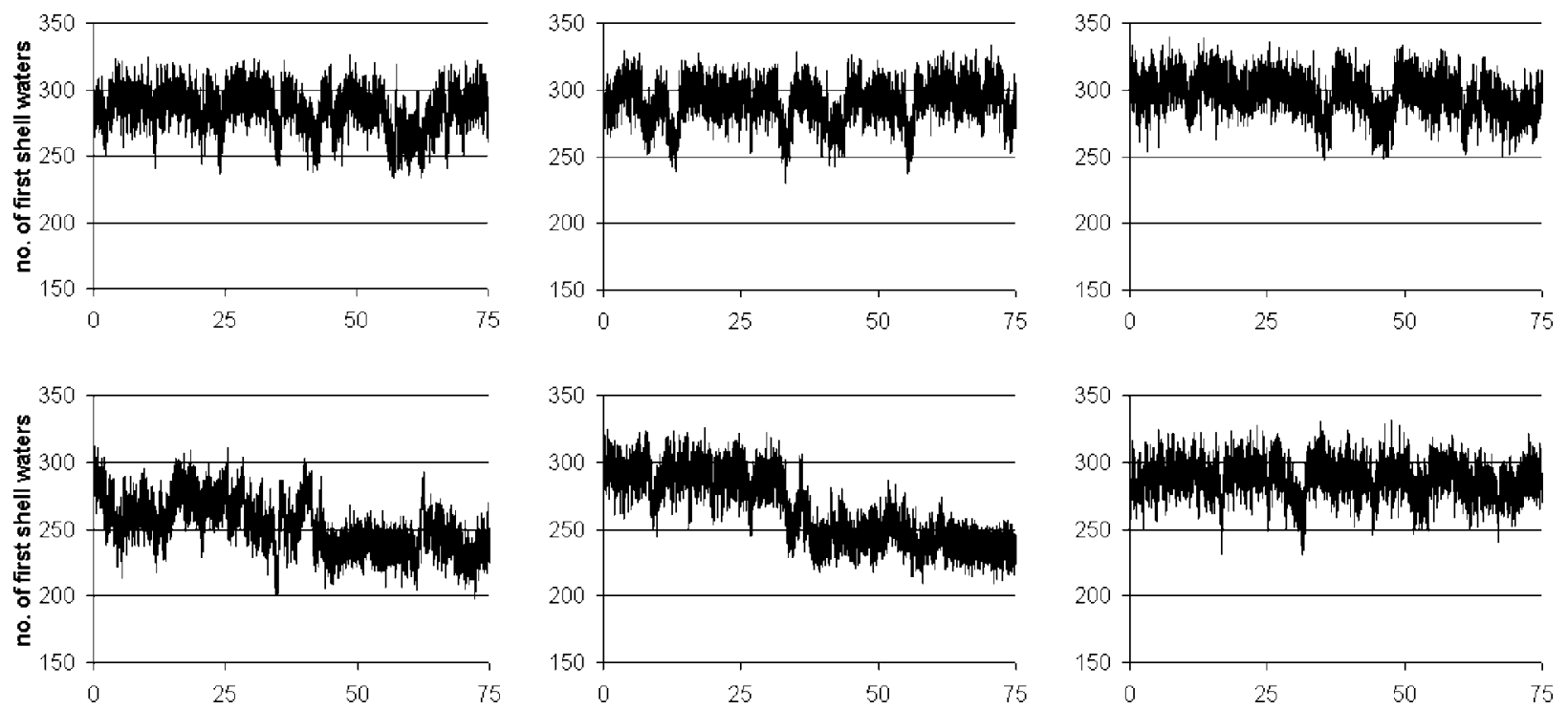

Figure 6. Time evolution of the total number of water molecules in the first hydration shell, i.e. within $3.5 \AA$ from solute atoms, for oN, oNM and oNL (from left to right) at $302 \mathrm{~K}$ (top panels) and $315 \mathrm{~K}$ (lower panels).
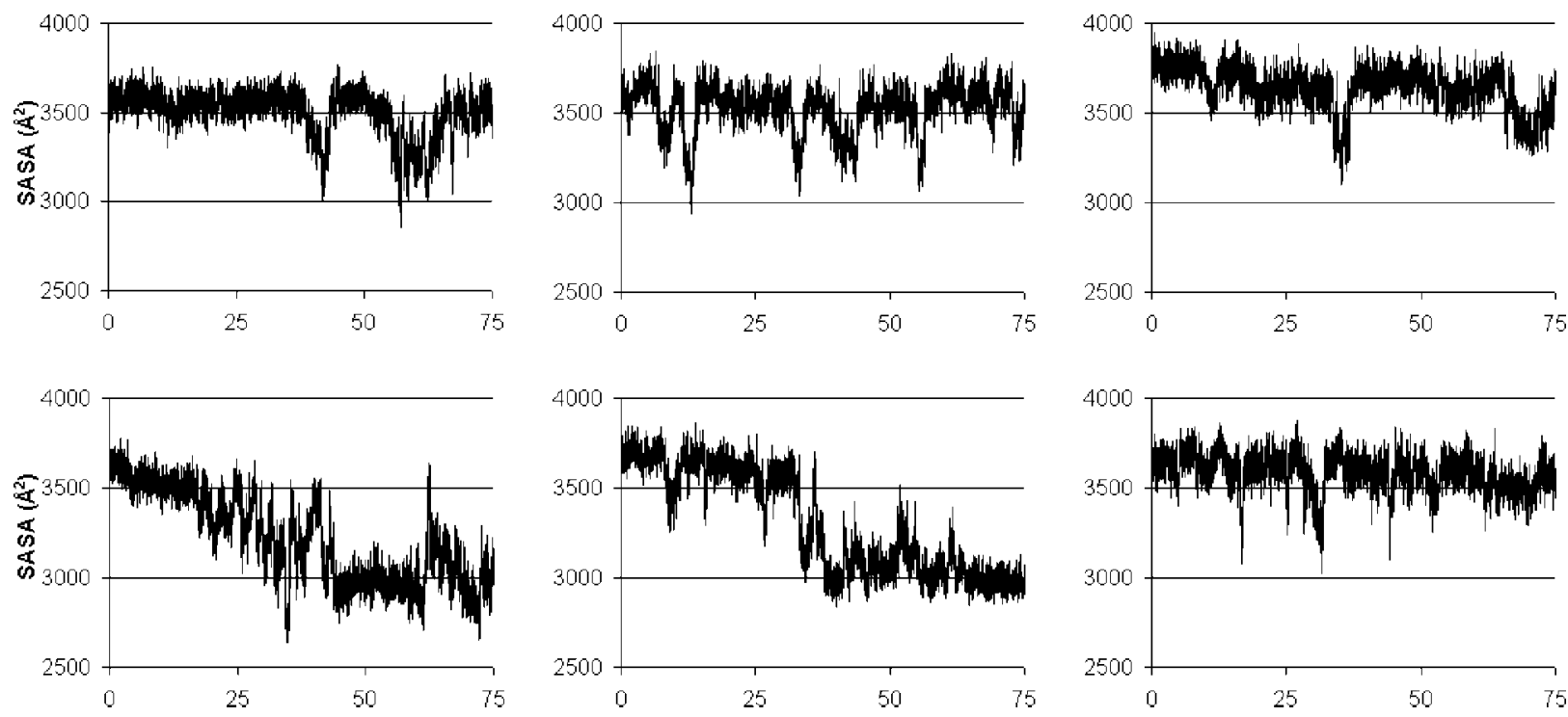

Figure 7. Time evolution of solvent accessible surface area (SASA) of the three oligomer molecules oN, oNM, and oNL (from left to right) at 302 $\mathrm{K}$ (top panels) and $315 \mathrm{~K}$ (lower panels).

all situations. The similarity of the behavior of the largest principal moments of inertia and of $R_{G}$ is explained by the relation, easily derived from the above definitions:

$$
M R_{G}^{2}=\frac{1}{2} \operatorname{Tr}((I))
$$

where $M$ is the total mass of the oligomer, Tr means trace of the inertia tensor and $R_{G}$ and $((I))$ are calculated on the same atoms. However the amount of information in $((I))$ is in principle more complete. ${ }^{25}$

Independent pieces of information are those relative to the number of water molecules within the first hydration shell of the solute, namely within $3.5 \AA$ from the solute atoms, (Figure 6 ) and to the solvent accessible surface $\operatorname{area}^{20}$ (SASA) (Figure 7). It is interesting to notice that almost all features evidenced in Figures 3-5 have a correspondence with the results of Figures 6 and 7. Indeed, looking at each single panel of the five Figures whenever there is a sudden change in one case, we find the same in the other four cases; analogously, when the behavior of one of the variables is stable on the average, the same happens in the others. The only difference is that, for the cases of oN and oNM at $315 \mathrm{~K}$ beyond $40 \mathrm{~ns}$, the observed values of SASA and number of water molecules still fluctuate remarkably around a constant average, while $R_{G}$, rmsd, and principal moments of inertia do not show similarly large fluctuations.

Among the possible causes for the observed phase transition for oN and oNM at $315 \mathrm{~K}$, we need to distinguish between those tied to the choice of the model and those independent of it. In the first series of events, we have observed, by direct inspection and by statistical analysis of backbone dihedrals, that a regular succession of syndiotactic residues of NIPAAm (dldldl...) strongly hinders conformational changes and favors linear backbone conformations, whereas isotacticity ...ddd ... or ...lll... favors the formation of kinks (see part 2 in the Supporting Information). We observe though that our synthesized model oligomers contain amounts of iso- and syndiotactic segments of various lengths, since we have no kind of information for tacticity on experimentally examined polymers. In any case we again point out that initial backbone geometry 

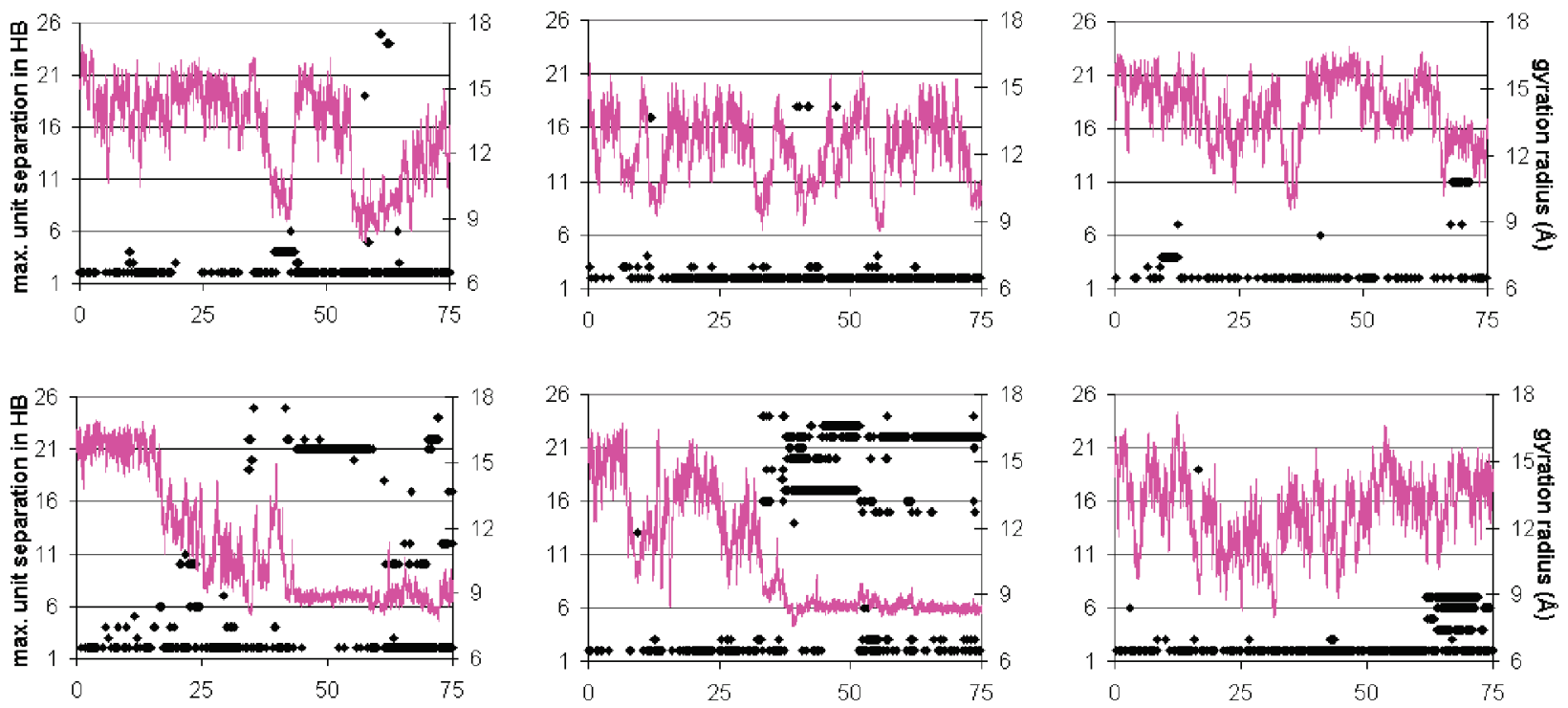

Figure 8. Superimposed time evolutions of the gyration radius $R_{G}$ (color) and of the maximum distance, in terms of number of units, between hydrogen bonded units, for oN, oNM, and oNL (from left to right) at $302 \mathrm{~K}$ (top panels) and $315 \mathrm{~K}$ (lower panels).
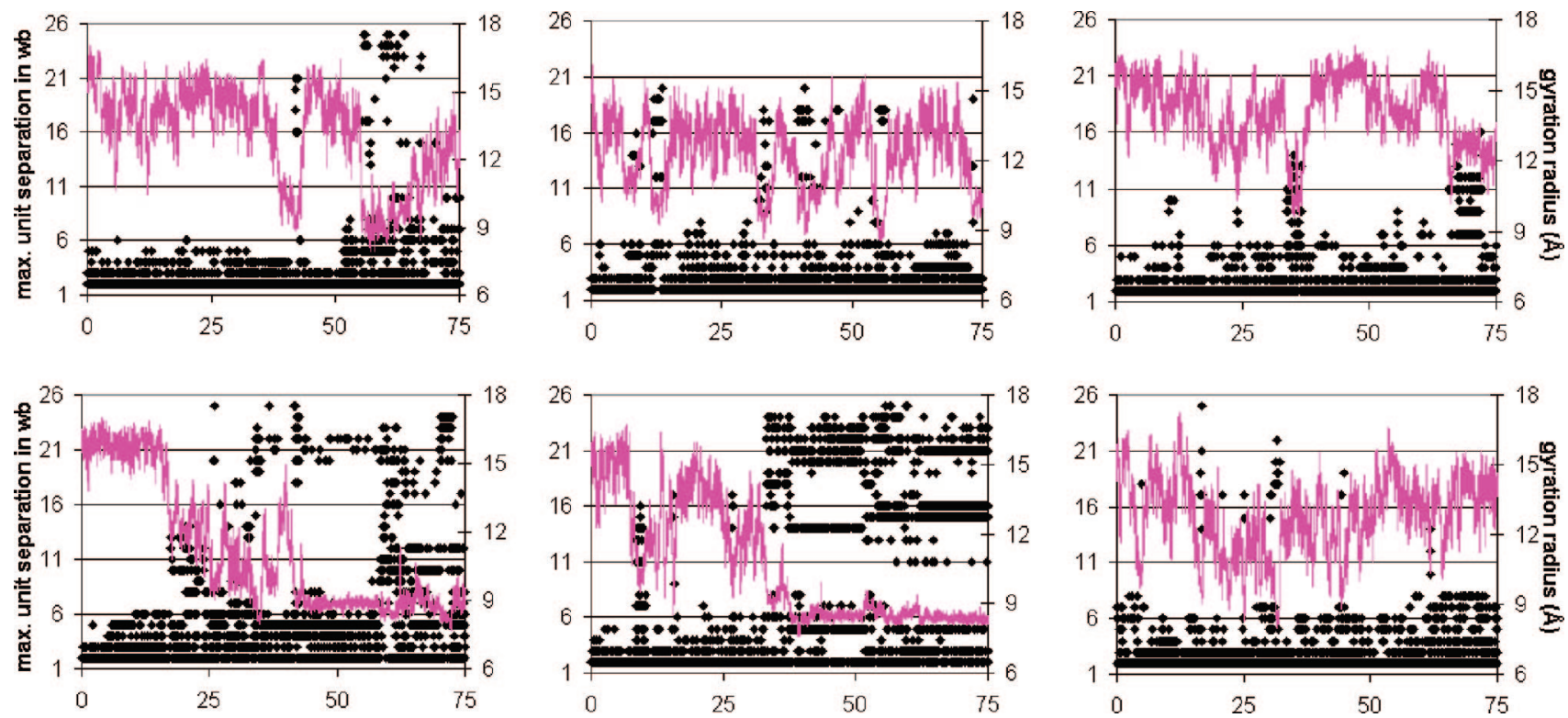

Figure 9. Superimposed time evolutions of the gyration radius $R_{G}$ (color) and of the maximum distance, in terms of number of units, between units connected by a one-water bridge, for oN, oNM and oNL (from left to right) at $302 \mathrm{~K}$ (top panels) and $315 \mathrm{~K}$ (lower panels).

is the same for all six simulations. For this reason we are confident that structural differences found at the end of them are determined by the interplay between the oligomers and the surrounding water molecules, that is to say by the organization of water initiated and progressively built by the establishment of either HBs or water- bridges of some solute's parts with others. To prove this, let us first look at Figures 8 and 9. In Figure 8, we show the time dependence of the maximum distance, in terms of number of units, between hydrogen bonded units. For comparison we give in the same Figure the corresponding time evolution of $R_{G}$. It is interesting to notice that, when $R_{G}$ decreases, even for short times, an intramolecular HB is very often formed between distant units. On the contrary, when the structure of the oligomer is close to the initial linear one, the distance of the HB-bonded units is quite small, possibly working against coiling. Finally and most importantly, at 315 $\mathrm{K}$ for oN and oNM, when the coiling is fully achieved, distant HBs are stable as well. Concurrent with this observation is the observation of the formation of water-bridges that link distant oligomer units: in Figure 9 we report the largest distances, in terms of unit numbers, between units connected by water-bridges involving just one water molecule. All observations made on the number of water molecules in the first hydration shell can be made also here, but probably the time behavior data in Figure 9 captures more incipient phenomena: in this way we observe, e.g., that the large structure fluctuations in oNL lead to large $R_{G}$ fluctuations and thus promote some water-bridges (and some intramolecular HBs) that, though, do not last for long enough times to promote a stable loop structure.

To better understand the mechanism of coiling, we have calculated for each oligomer unit the B-factor, according to the following expression:19

$$
B F(t)=(8 \pi / 3) \sum_{i}\left\langle\left|\mathbf{u}_{i}(t)\right|^{2}\right\rangle
$$

where $\mathbf{u}_{\mathrm{i}}(t)$ is the atomic displacement (for atom $i$ ) at time $t$, averaged over 10-ps time intervals. The importance of the B-factor (originally calculated as a preliminary step for the 

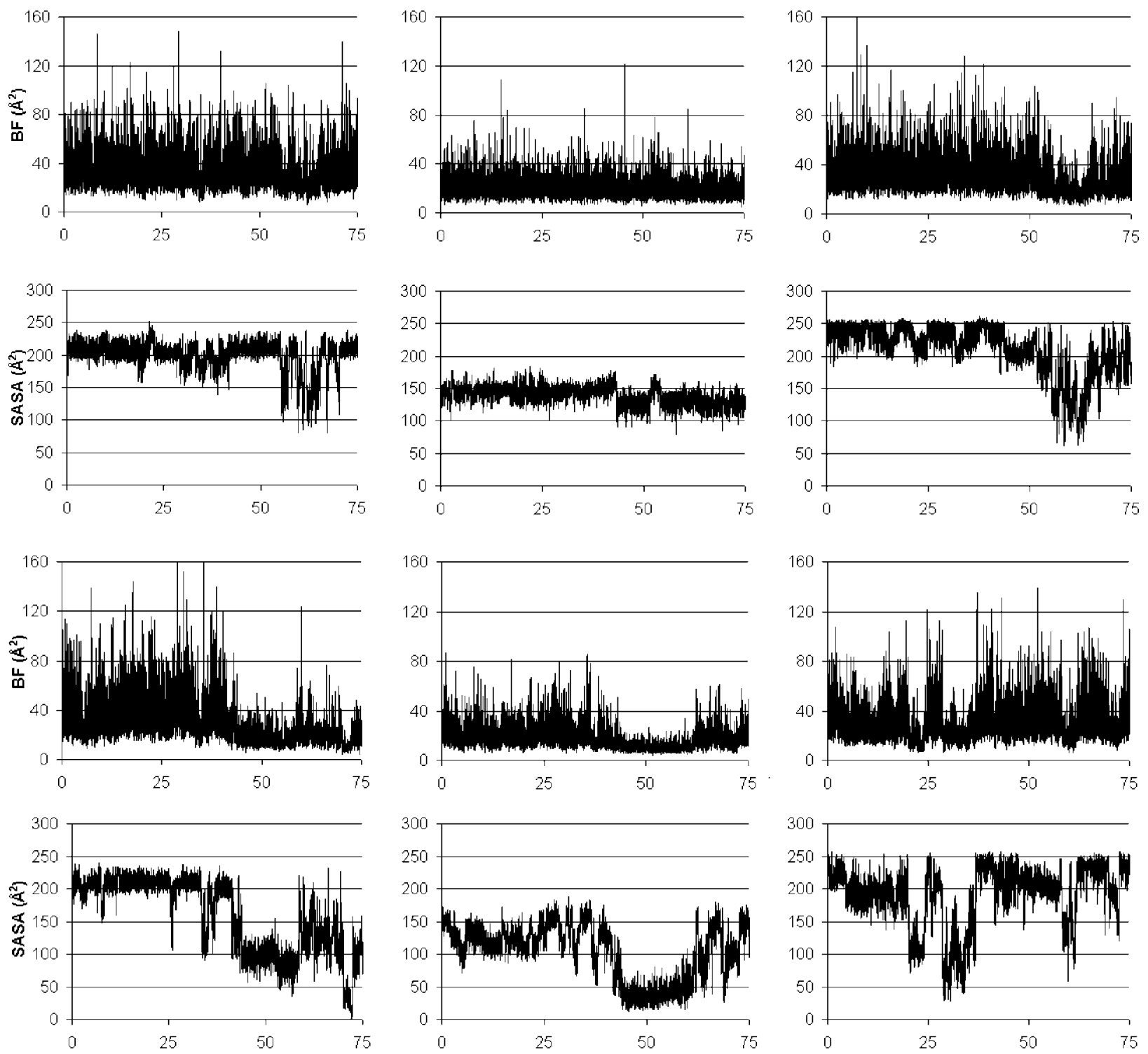

Figure 10. oN: time evolution of the B-factor for unit 1 (left), unit 22 (center) and unit 26 (right) (first and third rows), and of the corresponding SASA data (second and fourth rows) at $T=302 \mathrm{~K}$ (first two rows) and $T=315 \mathrm{~K}$ (last two rows).

evaluation of the Debye-Waller factor) has been recently evidenced to understand "useful" fluctuations leading given protein molecules to biological activity. ${ }^{26,27}$ We report below the results regarding few selected units, that are particularly involved in the transition from the linear to the coiled state at $315 \mathrm{~K}$. In Figure 10, we report $B F(t)$ data of oN vs time and correlate it to the corresponding SASA data for the terminal units 1 and 26 and for unit 22 at $302 \mathrm{~K}$ (top panels) and at $315 \mathrm{~K}$ (lower panels). Again the correspondence between the two quantities is striking, since it holds valid for so short time intervals as to be thought valid almost point by point. Besides the rather obvious result that internal unit 22 undergoes smaller oscillations than the terminal units, one may appreciate from the lower panels that when the transition to the coil occurs, both units 22 and 1 undergo smaller oscillation and expose themselves much less to water. This is related to the fact that the loop is formed by a direct correlation between units 1 and 22, as shown above also in Figures 8 and 9. Analogously, for oNM and oNL, in Figures 11 and 12, we report BF and SASA data for terminal unit 1 and units 9 and 19 bearing the charged and neutral MAVA (or MALEU) substitutions respectively. For oNM at $315 \mathrm{~K}$
(Figure 11 bottom), where a definite coiling is observed, the neutral unit 19 has a sudden decrease of BF and SASA values and this goes together with the parallel changes observed in unit 1; unit 9 instead, while bearing small oscillations, does not exhibit any change. The importance for loop formation of unit 19 may be related to the presence of a $\mathrm{COOH}$ group in it: indeed this provides to 19 , in respect to unit 9, an additional possibility of donor-type $\mathrm{HB}$, promoting its link to unit 1 . On the other hand we cannot exclude that the 1 to 19 distance be just about right for forming a stable loop, while the 1 to 9 distance may be too short. A propos of this, we have observed (data not reported here) that unit 9 preferably links to close by units 7 and 11 . At $302 \mathrm{~K}$ instead, no significant transition is observed for all the three units 1 , 9, and 19. This is also observed for oNL both at 302 and $315 \mathrm{~K}$ (Figure 12 top and bottom). We notice, by direct inspection of a VMD movie, that the behavior of unit 19 in oNL and oNM at $315 \mathrm{~K}$ bear some resemblance, even though the final fate of the two oligomers is different: what appears to be different is that unit 19 in oNL oscillates pointing outside the local oligomer bend formed by the adjacent units, while in oNM it oscillates pointing inside the loop. The latter 
behavior favors coiling, while the former one does not: the different behavior is possibly related to the unit 19 size, which is larger for MALEU than for MAVA comonomers.

In order to find possible causes of conformational rearrangements of the oligomers, we went back to our previous work, ${ }^{11}$ where we had shown by MD that, in vacuo, a p(NIPAAm) 50unit oligomer readily coils up, due to internal $\mathrm{HB}$ and/or Van der Waals forces; addition of water changes the scenario and $\mathrm{p}$ (NIPAAm) prefers to have a more extended conformation that favors hydration. This fact for $\mathrm{p}$ (NIPAAm) has been known for long time $\mathrm{e}^{5}$ and calls for a study of the properties of water surrounding the solute. A previous MD work ${ }^{28}$ on an alternative polymer exhibiting LCST, namely poly(ethylene oxide) (PEO), in presence of water, focused on the organization of water molecules' networks close and away from the solute molecules, generated by the presence of the latter ones. For this reason we have studied the self-diffusion coefficient of water molecules as a function of time, evaluated according to the Einstein relation: ${ }^{19}$

$$
D(t)=\frac{\left\langle(r(t+\Delta t)-r(t))^{2}\right\rangle}{6 \Delta t}
$$

where we average over the water molecules in a first shell surrounding the solute and the interval $\Delta t$ of observation is $10 \mathrm{ps} ; D(t)$ is evaluated by a linear regression on 20 steps in this interval. Some test calculations were performed dividing $\Delta t$ into 1000 steps instead of 20 and gave the same results, showing the reliability of the choice. In addition, in order to avoid fluctuations on time scales much smaller than the typical times of significant conformational changes, the obtained $D(t)$ values were averaged over $1 \mathrm{~ns}$ in two separate temporal regions of each simulation: the first one is chosen in the first $10 \mathrm{~ns}$ (where all the oligomers are almost linear); the second one is chosen around $40 \mathrm{~ns}$ where oN and oNM are coiled at $315 \mathrm{~K}$. The size of the first shell $r_{h}$ was varied, starting from the usual value of $r_{h}=3.5 \AA$ (i.e., for the first hydration shell) up to $r_{h}=30 \AA$, where all the water molecules in the box are included. The results of these calculations are reported in Figure 13.
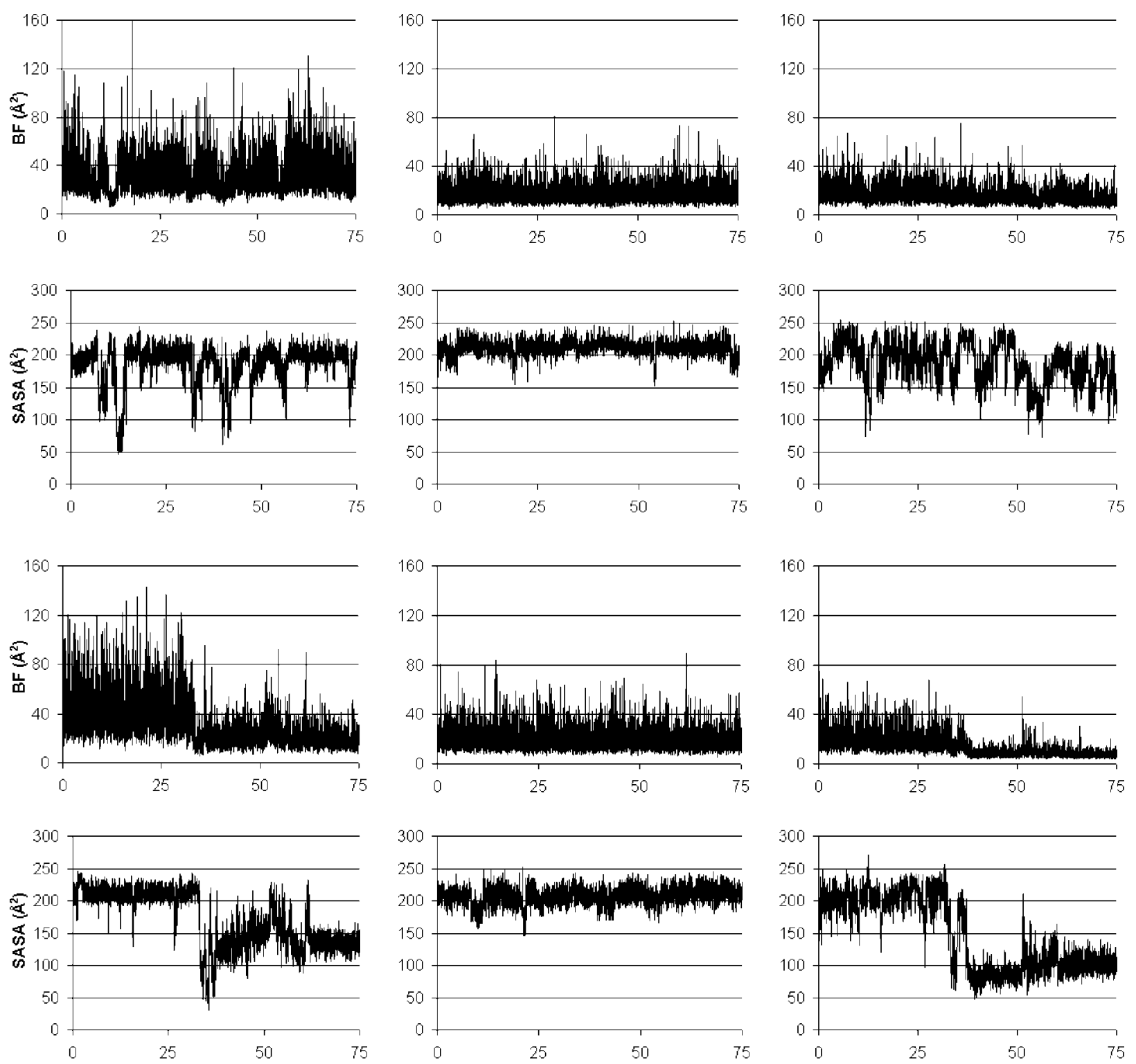

Figure 11. oNM: time evolution of the B-factor for unit 1 (left), unit 9 (center) and unit 19 (right) (first and third rows), and of the corresponding SASA data (second and fourth rows) at $T=302 \mathrm{~K}$ (first two rows) and $T=315 \mathrm{~K}$ (last two rows). 

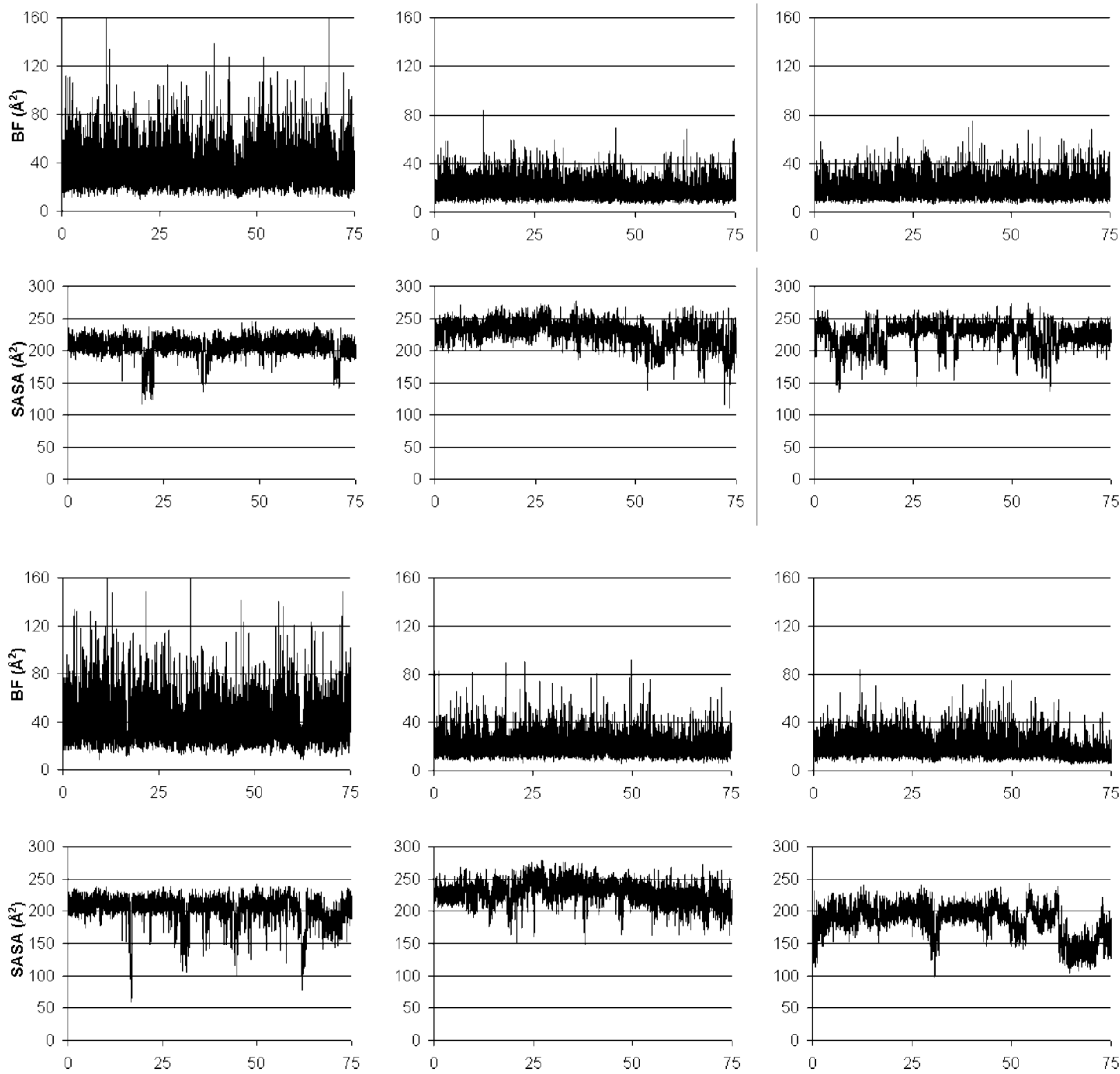

Figure 12. oNL: time evolution of the B-factor for unit 1 (left), unit 9 (center) and unit 19 (right) (first and third rows), and of the corresponding SASA data (second and fourth rows) at $T=302 \mathrm{~K}$ (first two rows) and $T=315 \mathrm{~K}$ (last two rows).
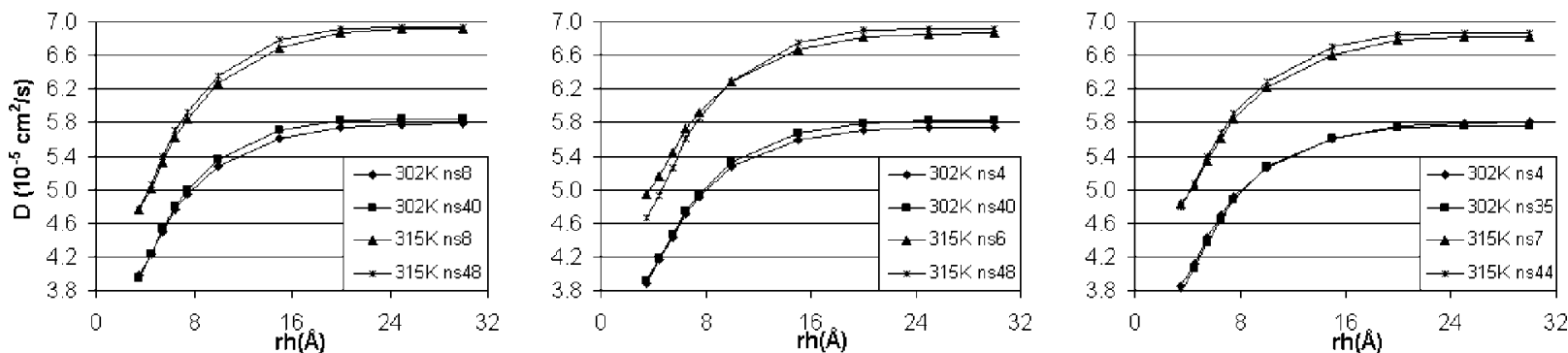

Figure 13. Self-diffusion coefficients of water $D\left(\right.$ in $10^{-5} \mathrm{~cm}^{2} / \mathrm{s}$ ) as function of the distance from the solute atoms: oN (left), oNM (center) and oNL (right) at $T=302 \mathrm{~K}$ and $T=315 \mathrm{~K} . D$ values are averaged over the following 1-ns time intervals: for oN 8th ns and 40th ns at $T=302 \mathrm{~K}$, 8th ns and 48th ns at $T=315 \mathrm{~K}$; for oNM 4th ns and 40th ns at $T=302 \mathrm{~K}$, 6th ns and 48th ns at $T=315 \mathrm{~K}$; for oNL 4th ns and 35th ns at $T=302$ $\mathrm{K}, 7$ th ns and 44 th ns at $T=315 \mathrm{~K}$.

Similar calculations for fewer water domains have been recently presented by Chiessi et al. ${ }^{29} \mathrm{We}$ observe that: $(i)$ for large water domains, the asymptotic $D$ values are close to those for bulk water at the same temperature, as expected. To further validate our results, we report in Table 2 the comparison of water self-diffusion coefficients $D$ calculated at large distances from oN, oNM, and oNL and of water alone; (ii) within smaller distances from the solute, calculated $D$ values are smaller, and this is related to water-solute interaction, including in particular the water-bridges between distant oligomer units (see Figure 9); hydrophobic interactions are also probably important, as shown in ref 29 for pVA, by comparing MD and neutron scattering data; (iii) the $D$ values for oN and oNM practically coincide, while they partly differ from those for oNL by a small 

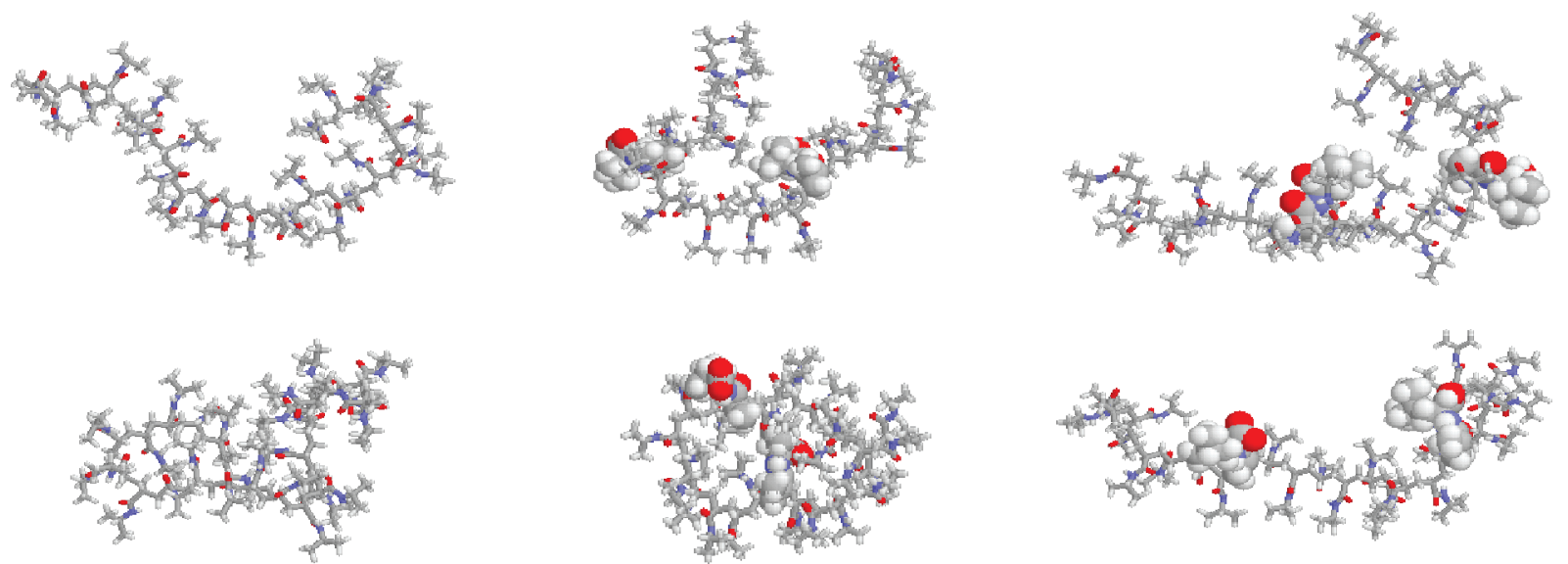

Figure 14. Conformations at $t=75 \mathrm{~ns}$ of the 26-unit oligomers oN, oNM, and oNL (from left to right) at $T=302 \mathrm{~K}$ (top row) and $T=315 \mathrm{~K}$ (bottom row). In the latter two cases the amino-acid-based substitutions are evidenced.

amount (see Table 2 and Figure 13). The latter aspect requires further investigation and may enlighten some features of the different phase transition taking place in p(NIPPAm-coMALEU) versus $\mathrm{p}$ (NIPAAm) and $\mathrm{p}($ NIPAAm- $c o$-MAVA).

\section{Conclusions}

With this work we have proved by MD simulation of water solutions and by statistical analysis of the trajectories, that at $315 \mathrm{~K}$ a 26-unit oligomer of NIPAAm $(\mathrm{oN})$ and a 26-unit oligomer obtained by replacing in oN two NIPAAm units with methacryloyl-l-valine (oNM), form stable coils in water, even though their initial conformations are linear with an all-trans backbone. Instead at the same temperature the oligomer obtained by replacing in oN two NIPAAm units with methacryloyl-l-leucine residues (oNL), does not form a stable loop, and its structure largely fluctuates. Representative pictures of the final structures for oN, oNM, and oNL are given in Figure 14, to be compared with the structures of Figure 2, reporting the initial conformations. This behavior of oNL prima facie seems to contradict the experimental results for $\mathrm{p}$ (NIPAAm-co-MALEU) reported in ref 8 since the observed LCST is lower compared to p(NIPAAm) and p(NIPAAm-co-MAVA). However in ref 8 [Figure 1], it is shown that the increase in overall scattering intensity for the MALEU-doped pNIPAAm, related to large intermolecular interactions, begins at lower temperatures $(\sim 298 \mathrm{~K}$, in correspondence with a sharp decrease of the hydrodynamic radius) and lasts for a larger $T$-interval, $\sim 7 \mathrm{~K}$. On the contrary p(NIPAAm-co-MAVA) aggregation occurs more suddenly in a $2 \mathrm{~K}$ interval starting at $\sim 305 \mathrm{~K}$, while coiling of single chains starts at lower temperature as evidenced by hydrodynamic radius data. The present MD results indicate that, while oNL oligomer undergoes, in both temperature regimes, large fluctuations and never reaches a compact form, oN and oNM form compact structures at higher temperature and, therefrom, fluctuate considerably less. From this we infer that the large oscillations in segments of p(NIPAAm-co-MALEU) may favor intermolecular interactions and, consequently, promote slow interchain aggregation, while the compact form does not. ${ }^{8}$ On the other hand any comparison with experiments should be carried on, taking into account the limits of modeling and calculations, i.e. we have dealt with a short oligomer, and we have not treated more than one chain.

However we feel we have studied in depth the solute-water interaction: in particular, a large part of the statistical analysis of the results, presented in the previous section, has been devoted to the role played by intramolecular HB and waterbridges in the oligomer structural changes. The water-solute interaction has been in particular assayed by investigating the water self-diffusion coefficient, which has been found to characteristically depend on the distance from the solute oligomers.

Supporting Information Available: Part 1, a table of Cartesian coordinates of the three monomers and, part 2, text giving a statistical analysis of the time evolution of the dihedral angles of the backbone and a figure showing their relationship to the tacticity of the chains. This material is available free of charge via the Internet at http://pubs.acs.org.

\section{References and Notes}

(1) Heskins, M.; Guillet, J. E. J. Macromol. Sci. Chem. 1968, A2, 1441.

(2) Schild, M. G. Prog. Polym. Sci. 1992, 17, 163.

(3) Hoffman, A. S. Clin. Chem. 2000, 46, 1478.

(4) Aguilar, M. R.; Elvira, C.; Gallardo, A.; Vàzquez, B.; Romàn, J. Smart Polymers and Their Applications, in E-book: Topics in Tissue Engineering, Volume 3, 2007, Eds. Ashammakhi, N.; Reis, R.; Chiellini, E.

(5) (a) For the LCST definition, see for example: Taylor, L. D.; Ceranowski, L. D. J. Polym. Sci., Polym. Chem. Ed. 1975, 13, 25512570. (b) Schild, M. G.; Tirrel, D. A. J. Phys. Chem. 1990, 94, 43524356.

(6) Rička, J.; Mewes, M.; Nyffenegger, R.; Binkert, Th. Phys. Rev. Lett. 1990, 65, 657.

(7) Wu, C.; Wang, X. Phys. Rev. Lett. 1998, 80, 4092.

(8) Lebon, F.; Caggioni, M.; Bignotti, F.; Abbate, S.; Gangemi, F.; Longhi, G.; Mantegazza, F.; Bellini, T. J. Phys. Chem. B 2007, 111, 2372.

(9) Casolaro, M. Macromolecules 1995, 28, 235.

(10) Liu, R. G. W.; Cantin, S.; Perrot, F.; Winnik, F. M. Polym. Adv. Technol. 2006, 17, 798-803.

(11) Longhi, G.; Lebon, F.; Abbate, S.; Fornili, S. L. Chem. Phys. Lett. 2004, 386, 123.

(12) Lebon, F.; Bignotti, F.; Penco, M.; Gangemi, R.; Longhi, G.; Abbate, S. Chirality 2003, 15, 251.

(13) See for example: Leach, A. R. Molecular Modelling. Principles and Application; 2nd ed., Prentice Hall: Upper Saddle River, NJ, 2001.

(14) GAUSSIANO3; Gaussian Inc.: Pittsburgh, PA; www.gaussian.com

(15) Bayly, C. I.; Cieplak, P.; Cornell, W. D.; Kollman, P. A. J. Phys. Chem. 1999, 97, 10269.

(16) Case, D. A.; Pearlman, D. A.; Caldwell, J. W.; Cheatham, T. E., III.; Wang, J.; Ross, W. S.; Simmerling, C. L.; Darden, T. A.; Merz, K. M.; Stanton, R. V.; Cheng, A. L.; Vincent, J. J.; Crowley, M.; Tsui, V.; Gohlke, H.; Radmer, R. J.; Duan, Y.; Pitera, J.; Massova, I.; Seibel, G. L.; Singh, U. C.; Weiner, P. K.; Kollman, P. A. AMBER 7; University of California: San Francisco, CA, 2002.

(17) Jorgensen, W. L.; Chandrasekhar, J.; Madura, D.; Impey, R. W.; Klein, M. L. J. Chem. Phys. 1983, 79, 926.

(18) Duan, Y.; Wu, C.; Chowdhury, S.; Lee, M. C.; Xiong, G. M.; Zhang, W.; Yang, R.; Cieplak, P.; Luo, R.; Lee, T.; Caldwell, J.; Wang, J. M.; Kollman, P. J. Comput. Chem. 2003, 24, 1999. 
(19) Allen, M. P.; Tildesley, T. J. Computer Simulation of Liquids; Clarendon Press: Oxford, U.K., 1987.

(20) Hubbard, S. J.; Thornton, J. M. "NACCESS", Computer Program; Department of Biochemistry and Molecular Biology, University College: London, U.K.

(21) Bergman, D. L.; Laaksonen, L.; Laaksonen, A. J. Mol. Graph. Model. 1997, 15, 301.

(22) Sayle, R. A.; Milner-Whilte, E. J. Trends Biochem. Sci. 1995, 20, 374

(23) Humphrey, W.; Dalke, A.; Schulten, K. J. Mol. Graphics 1996, $14,33$.

(24) McDonald, I. K.; Thornton, J. M. J. Mol. Biol. 1994, 238, 777793.
(25) Catte, A.; Patterson, J. C.; Bashtovyy, D.; Jones, M. K.; Gu, F.; Li, L.; Rampioni, A.; Sengupta, D.; Vuorela, T.; Niemelä, P.; Kartunen, M.; Marrink, S. J.; Vattulainen, I.; Segrest, J. P. Biophys. J. 2008, 94, 23062319.

(26) Frauenfelder, H. Intl. J. Quantum Chem. 1989, XXXV, 711-715.

(27) Hamacher, K.; McCammon, J. A. J. Chem. Theory Comput. 2006, $2,873-878$.

(28) Smith, G. D.; Bedrov, D.; Borodin, O. Phys. Rev. Lett. 2000, 26, $5583-5586$.

(29) Chiessi, E.; Cavalieri, F.; Paradossi, G. J. Phys. Chem. B, 2007, $111,2820-2827$.

JP803545P 